www.jmscr.igmpublication.org

Impact Factor 5.84

Index Copernicus Value: 83.27

ISSN (e)-2347-176x ISSN (p) 2455-0450

crossref DOI: _https://dx.doi.org/10.18535/jmscr/v5i3.130

Journal Of Medical Science And Clinical Research

IGM Publication

An Official Publication of IGM Publication

\title{
Comparison of Scoring Systems for Mortality Prediction in Acute Exacerbation of Chronic Obstructive Pulmonary Disease
}

\author{
Authors \\ Dr Neethu Thambi, Dr K V Padmanabhan, Dr Manoj D K, Dr Rajani M. \\ Department of Respiratory Medicine, Academy of Medical Sciences, Pariyaram, Kannur, Kerala \\ Correspondence Author
}

Dr Neethu Thambi

Junior Resident, ACME, Pariyaram, Kannur, Kerala

Email: ofneethu@gmail.com, Phone: 9744658925

\begin{abstract}
Acute exacerbation of chronic obstructive pulmonary disease (AECOPD) is a frequent cause of emergency admission. A scoring system is invaluable in predicting mortality and guiding therapeutic interventions.

Aims: Comparison of DECAF, BAP-65, CURB-65, CAPS, APACHE II risk scores in predicting in hospital mortality in AECOPD.

Methods: 150 patients of AECOPD, admitted during ten month period were scored at admission using all 5 scores and their ability to predict in-hospital mortality was analysed. The cut off value of scores that best predicted mortality was identified. Individual parameters that correlated best with mortality were also documented.

Results: DECAF score $>=2$, with an AUROC of 0.729, correlated best with mortality. APACHE 2 score $>=18$ could predict in hospital mortality with an AUROC of 0.700, CURB-65>=2 predicted mortality with an AUROC of 0.709.Parameters that were most closely associated with mortality were Altered Mentation, Respiratory rate $>30 /$ min, Hyponatremia $(S . N a+<130 m E q / L$, Hypoalbuminemia $(<3.5 \mathrm{~g} / \mathrm{dL})$.

Conclusion: DECAF score( $>=2)$ best correlates with in hospital mortality. Individual parameters of Altered Mentation(GCS<15), Respiratory rate $>30 / \mathrm{min}$, Baseline dyspnoea of eMRCD 5b, Elevated WBC count (>40000), Hyponatremia (S.Na+<130mEq/L, Hypoalbuminemia $(<3.5 \mathrm{~g} / \mathrm{dL})$ correlates best with mortality.

Keywords: acute exacerbation, COPD, DECAF, CAPS, APACHE II, CURB 65, BAP.
\end{abstract}

\section{INTRODUCTION}

Acute exacerbations of chronic obstructive pulmonary disease (AECOPD) is defined as "An acute worsening of respiratory symptoms that result in additional therapy"1. Patients in exacerbation face the immediate risks of respiratory failure and death and in the long-term it results in impaired lung function, poor prognosis and increased risk of mortality. Most patients with AECOPD visiting Emergencies have severe exacerbation and need hospitalization, may require Intensive care and mechanical ventilation if respiratory failure is present. In such situations, assessing the severity of Exacerbation is mandatory to guide decisions regarding degree of care (home, hospital ward or intensive care unit) as well as intensity of, treatment, initiation of mechanical ventilation and follow-up. ${ }^{2}$ 
A large number of scores have been developed for AECOPD like DECAF ${ }^{3}$, modified DECAF, CAPS $^{4}$. Scores like CURB- $65^{5}$ which was originally developed for pneumonia has also been used in AECOPD, and general ICU scoring systems like APACHEII ${ }^{6}$ has been used to predict mortality. Many studies comparing these systems have also been done on various populations with different results ${ }^{2,7,8}$.

In order to recommend a score for use in the Emergency room, a comparison of the performance of these scores in the present setting had to be done. We selected five scoring systemsDECAF, CAPS, BAP, CURB-65, APACHE II which were based on routinely measured parameters, and compared their performance in predicting in hospital mortality.

\section{AIM}

To compare performance of five risk scores (DECAF, BAP, CURB-65, COPD and Asthma Physiology Score (CAPS), Acute Physiology and Chronic Health Evaluation II (APACHE II)) in AECOPD and to find the score that best predicts in hospital mortality.

\section{MATERIALS AND METHODS}

A hospital based prospective study was conducted in The Department of Respiratory medicine for ten month period.

Study population was a consecutive sample of 150 patients admitted with AECOPD during the study period.

Inclusion criteria- Previously diagnosed COPD, Age $>40$, Admitted with primary diagnosis of AECOPD

Exclusion criteria- Other primary cause of present admission, Myocardial Infarction(MI), Chronic Kidney Disease(CKD), Acute Kidney Injury(AKI) \& Malignancy, unwilling to participate, Left/ Referred without completing treatment Informed consent taken. Detailed history including age, sex, smoking, and assessment of stable state dyspnea grade over the preceding 3 months based on the extended Medical Research
Council Dyspnea Score (eMRCD) ,clinical examination including assessment of mental state conscious level and signs of severity of exacerbation (cyanosis, use of accessory inspiratory muscles, paradoxical abdominal movement, asterixis, neurological impairment, lower limb edema), chest radiological examination, ECG, arterial blood gases analysis, measurement of blood urea nitrogen (BUN), complete blood count (CBC). Severity scores for AECOPD were calculated for each patient and the individual parameters used in calculating the scores were also recorded.

DECAF has 5 variables (Baseline dyspnoea eMRCD $5 \mathrm{a}$ or $5 \mathrm{~b}$, Eosinopenia $\left(<0.05^{*} 10^{3} / \mu \mathrm{L}\right)$, Consolidation, Acidemia $(\mathrm{PH}<7.3)$, Atrial Fibrillation), a maximum score of 6. BAP score uses 4 variables(Elevated BUN, Altered Mental Status, Pulse Rate $>109 / \mathrm{min}$, Age $>65$ ) maximum score of 4; CURB65 uses 5 variables Confusion, Blood Urea $>45 \mathrm{mg} / \mathrm{dL}$, Respiratory Rate $>=30$, $\mathrm{SBP}<90$ or $\mathrm{DBP}<60$, Age $>65$ ) and is scored out of 5. CAPS uses 7 variables scored differentially according to their value (Heart Rate, Mean Arterial Pressure, PH, Sodium, Urea, Creatinine, Albumin, WBC count) maximum score of 100. APACHE II is scored out of a maximum of 71 based on 12 physiologic variables, age, and chronic health.

Patients were admitted and managed according to their condition and current hospital protocols, prognosis was recorded, as either recovery and discharge or death.

\section{STATISTICAL ANALYSIS}

Data was analysed with Spss v24. Receiver operator curves(ROC) analysis performed. Area under ROC(AUROC) indicates the accuracy of a diagnostic score. AUROC of $>0.7$ indicates fair accuracy. The scoring system with the largest AUROC was selected. A cut off score with highest sensitivity and specificity selected.

Relationship between 17 individual parameters (which were used to derive the scores) and mortality analysed with chi square test. 


\section{JMSCR Vol||05||Issue||03||Page 19125-19132||March}

Parameters which were associated with mortality at a significance level of 0.01 identified.

\section{RESULTS}

The study population consisted of 150 consecutive patients. $128(85.3 \%)$ were males and $22(14.7 \%)$ females. 130 out of 150 were current or ex smokers, which included 125 out of the 128 males and 5 out of the 22 females. Average age of study group was 66.1 (SD8.3).

95 patients required admission to a critical care unit, 62 patients had acidemia with an average $\mathrm{PH}$ of $7.18(\mathrm{SD}=0.12) .41$ of these patients needed invasive mechanical ventilation and they remained on ventilator for an average of 4.71 days ( $\mathrm{SD}=2.8)$. Average hospital stay was 4.8 days $(\mathrm{SD}=1.88)$ for those admitted in ward, 6.4 days $(\mathrm{SD}=2.5)$ for those admitted to ICU. 38 of the 150 patients died in hospital, resulting in a $25 \%$ mortality, 6 of these patients were initially admitted to ward.

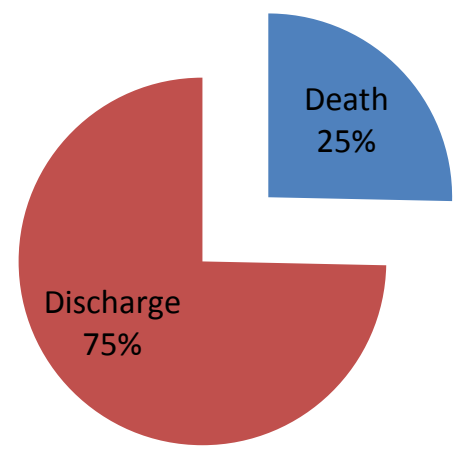

Graph 1: Mortality in study population

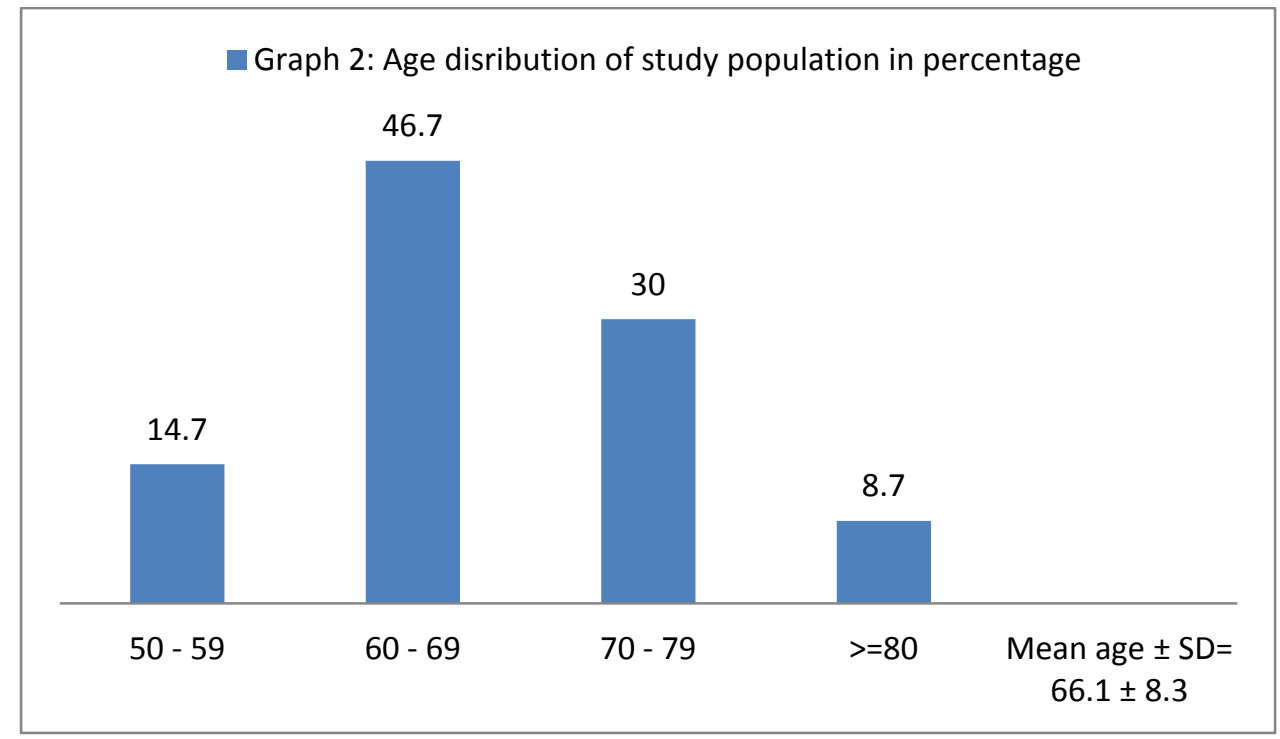

Graph 2: Age distribution of study population

Various individual parameters and their correlation with in hospital mortality was analysed with $\chi^{2}$ test. The parameters that were found to be significant at 0.01 level were Altered Mentation $(\mathrm{GCS}<15), \quad$ Respiratory rate $>30 / \mathrm{min}$, Baseline dyspnoea of eMRCD 5b, Elevated WBC count (>40000), Hyponatremia (S.Na+ <130mEq/L, Hypoalbuminemia $(<3.5 \mathrm{~g} / \mathrm{dL})$ at the time of admission. 
Table 1: Comparison of various parameters based on outcome

\begin{tabular}{|c|c|c|c|c|c|c|c|c|c|c|c|}
\hline & \multirow{3}{*}{\multicolumn{3}{|c|}{ Parameters }} & \multicolumn{6}{|c|}{ Outcome } & \multirow{3}{*}{$\chi^{2}$} & \multirow{3}{*}{$\mathrm{p}$} \\
\hline & & & & \multicolumn{3}{|c|}{ Death } & \multicolumn{3}{|c|}{ Discharge } & & \\
\hline & & & & \multicolumn{2}{|c|}{ Count } & Percent & \multicolumn{2}{|c|}{ Count } & Percent & & \\
\hline 1 & \multicolumn{3}{|c|}{ Eosinopenia $\left(<0.05 * 10^{3} / \mu \mathrm{L}\right)$} & \multicolumn{2}{|c|}{23} & 33.8 & \multicolumn{2}{|c|}{45} & 66.2 & $4.74 *$ & 0.029 \\
\hline 2 & \multicolumn{3}{|c|}{ Consolidation } & \multicolumn{2}{|c|}{12} & 32.4 & \multicolumn{2}{|c|}{25} & 67.6 & 1.31 & 0.253 \\
\hline 3 & \multicolumn{3}{|c|}{ Acidemia $(\mathrm{PH}<7.3)$} & \multicolumn{2}{|c|}{20} & 32.3 & \multicolumn{2}{|c|}{42} & 67.7 & 2.68 & 0.102 \\
\hline 4 & \multicolumn{3}{|c|}{ Atrial Fibrillation } & \multicolumn{2}{|c|}{0} & 0.0 & \multicolumn{2}{|c|}{9} & 100.0 & 3.25 & 0.071 \\
\hline 5 & \multicolumn{3}{|c|}{ Exacerbations $(>2 / \mathrm{yr})$} & \multicolumn{2}{|c|}{25} & 24.0 & \multicolumn{2}{|c|}{79} & 76.0 & 0.3 & 0.584 \\
\hline 6 & \multicolumn{3}{|c|}{ Elevated BUN } & \multicolumn{2}{|c|}{18} & 33.3 & \multicolumn{2}{|c|}{36} & 66.7 & 2.85 & 0.091 \\
\hline 7 & \multicolumn{3}{|c|}{ Altered Mentation } & \multicolumn{2}{|c|}{17} & 44.7 & \multicolumn{2}{|c|}{21} & 55.3 & $10.13 * *$ & 0.001 \\
\hline 8 & \multicolumn{3}{|c|}{ Tachycardia (>109/min) } & 2 & & 34.4 & 4 & & 65.6 & $4.82 *$ & 0.028 \\
\hline 9 & AGE $>65$ & & & 2 & & 26.6 & 6 & & 73.4 & 0.21 & 0.645 \\
\hline 10 & $\mathrm{RR}>30 / \mathrm{min}$ & & & 2 & & 49.2 & 3 & & 50.8 & $29.17 * *$ & 0.000 \\
\hline 11 & Hypotension & $\mathrm{P}<90 / 60 \mathrm{~m}$ & & 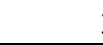 & & 50.0 & 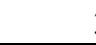 & & 50.0 & 2.01 & 0.156 \\
\hline 12 & Hyponatremi & $<130 \mathrm{mEq}$ & & 3 & & 33.3 & 7 & & 66.7 & $16.07 * *$ & 0.000 \\
\hline 13 & Hypoalbumir & $\operatorname{nia}(<3,5 \mathrm{~g} /$ & & 1 & & 50.0 & & & 50.0 & $13.08 * *$ & 0.000 \\
\hline 14 & & $<14000$ & & & & 19.5 & 7 & & 80.5 & & \\
\hline & & $(14000-$ & & 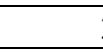 & & 11.1 & 2 & & 88.9 & $2034 * *$ & 0000 \\
\hline & WBC count & ( 20000 - & 99) & & & 45.5 & & & 54.5 & $20.34 * \pi$ & 0.000 \\
\hline & & $(>40000$ & & 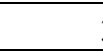 & & 100.0 & 7 & & 0.0 & & \\
\hline 15 & & Nil & & & & 14.6 & 7 & & 85.4 & & \\
\hline & Baseline & eMRCD & $5 \mathrm{a}$ & 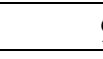 & & 23.1 & & & 76.9 & & \\
\hline & Dyspnoea & Grade & $5 b$ & & & 72.7 & & & 27.3 & $31.64 * *$ & 0.000 \\
\hline & & & & Mean & SD & $\mathrm{N}$ & Mean & SD & $\mathrm{N}$ & $\chi^{2}$ & $\mathrm{p}$ \\
\hline 16 & Mean arteria & ressure $(\mathrm{m}$ & $\mathrm{fg})$ & 91.1 & 14.4 & 38 & 94.7 & 14.6 & 112 & 1.33 & 0.187 \\
\hline 17 & Urea $(\mathrm{mg} / \mathrm{dL}$ & & & 43.9 & 25.8 & 38 & 33.7 & 22.5 & 112 & $2.32 *$ & 0.022 \\
\hline 18 & Creatinine $(\mathrm{m}$ & & & 2.0 & 1.5 & 38 & 1.3 & 0.9 & 112 & $3.26 * *$ & 0.001 \\
\hline
\end{tabular}

Predictive power of different scoring systems

\section{for in hospital mortality}

Area under (ROC) curve is a summary measure of the accuracy of a quantitative diagnostic test. Two or more diagnostic tests can be compared by the difference in AUC. An AUROC of $>0.70$ indicates a fairly accurate test. The largest area under ROC was 0.729 for DECAF, followed by CURB-65 with 0.709 and APACHE 2 which had 0.700. CAPS score had an AUROC of 0.680 and BAP score had 0.668 , which indicated they had poor accuracy in this setting. Comparing the AUROC of DECAF with CURB-65 \& APACHE2 , the differences were not statistically significant.

\section{Graph 3: ROC curve for DECAF}

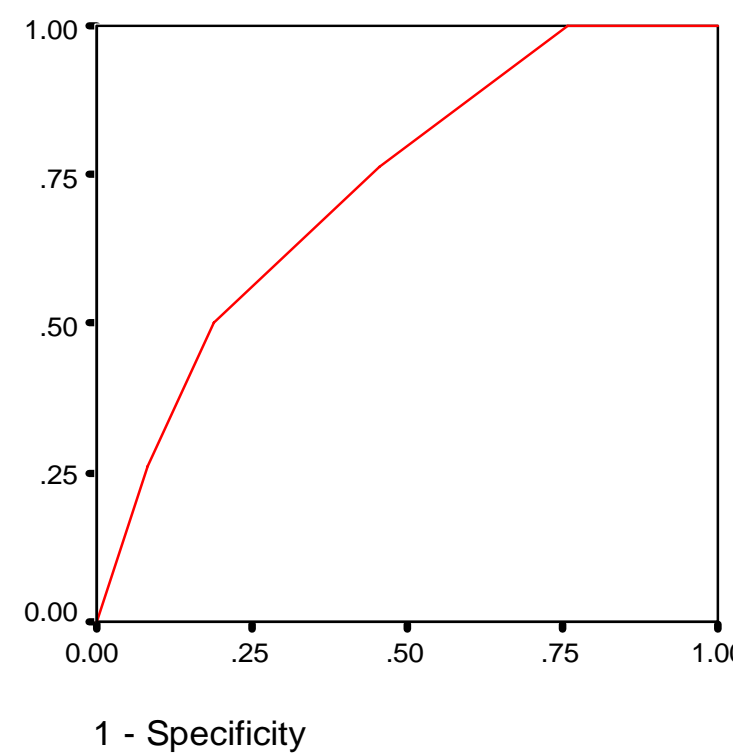

Diagonal segments are produced by ties. 
Graph 4: ROC curve for BAP

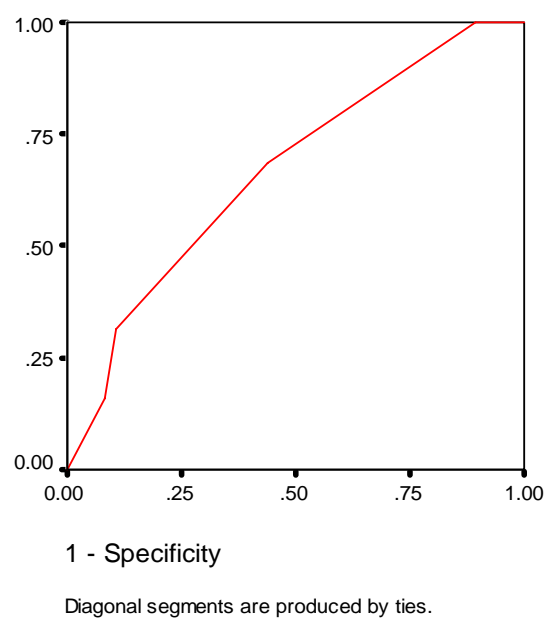

Area Under the curve $=0.668$

Graph 5: ROC curve for CURB65

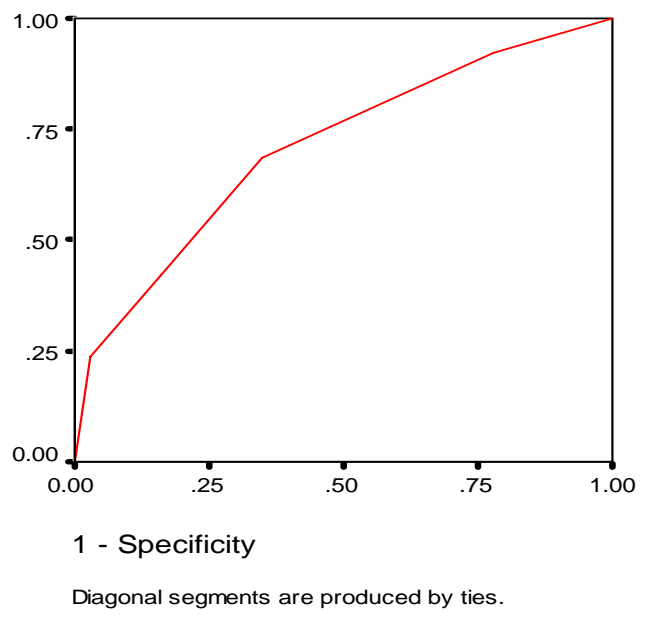

Area Under the curve $=0.709$

Graph 6: ROC curve for prediction of CAPS

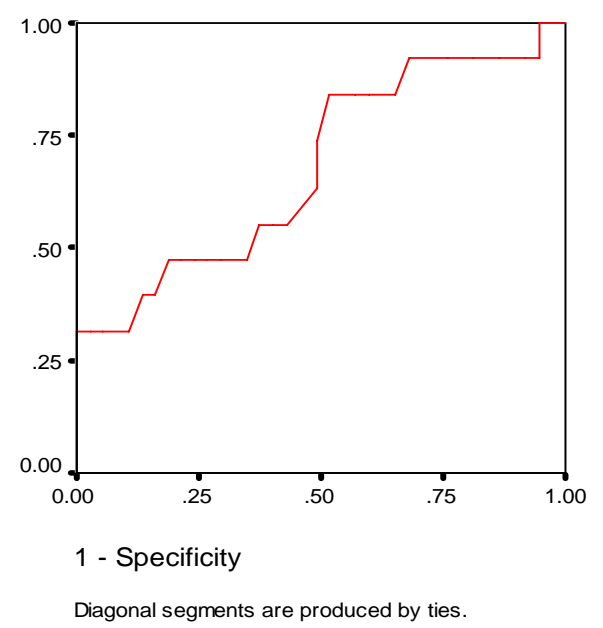

Area Under the curve $=0.680$
Graph 7: ROC curve for prediction of APACHE II

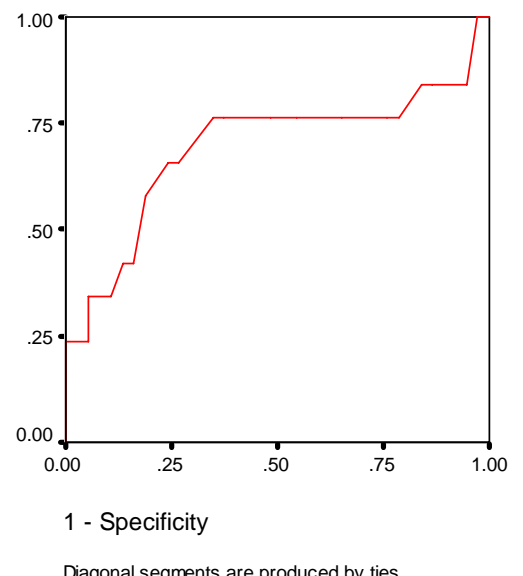

Area Under the curve $=0.700$

\section{Graph 8: Difference In Area Under ROC}

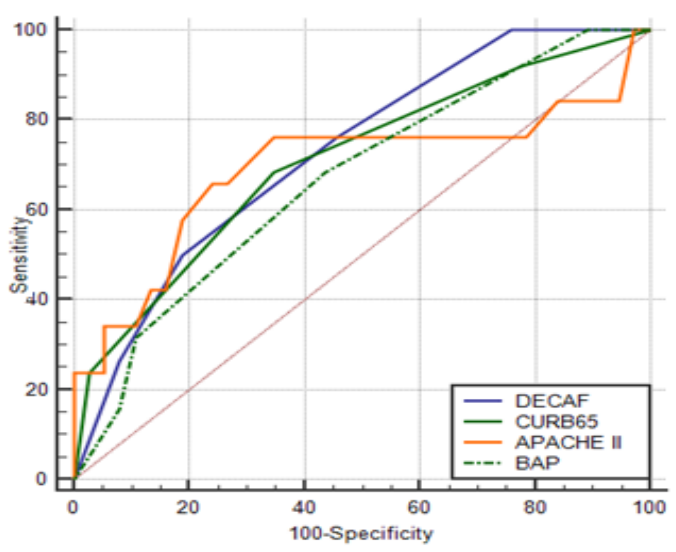

\begin{tabular}{|l|c|c|c|}
\hline \multicolumn{4}{|c|}{ Table 2: AUROC Of Scoring Systems } \\
\hline Variable & AUC & $\mathbf{S E}^{\mathbf{a}}$ & $\mathbf{9 5 \%} \mathbf{C I}^{\mathbf{b}}$ \\
\hline DECAF & 0.729 & 0.0431 & 0.651 to 0.799 \\
\hline BAP & 0.668 & 0.0469 & 0.587 to 0.743 \\
\hline CURB65 & 0.709 & 0.0489 & 0.630 to 0.780 \\
\hline CAPS & 0.680 & 0.0531 & 0.599 to 0.754 \\
\hline APACHE-II & 0.700 & 0.0601 & 0.612 to 0.765 \\
\hline
\end{tabular}

${ }^{a}$ DeLong et al., 1988

${ }^{\mathrm{b}}$ Binomial exact

\begin{tabular}{|l|c|c|c|}
\hline \multicolumn{4}{|l|}{ Table 3: Comparison of AUROC } \\
\cline { 1 - 3 } SCORE & CUT OFF & $\underline{\mathbf{A U R O C}}$ & \multirow{2}{*}{ VS DECAF P $=$} \\
\hline DECAF & $>=\mathbf{2}$ & $\underline{\mathbf{0 . 7 2 9}}$ & \\
\hline CURB-65 & $>=2$ & 0.709 & 0.74 \\
\hline APACHE 2 & $>=18$ & 0.700 & 0.63 \\
\hline CAPS & $>=31$ & 0.680 & 0.46 \\
\hline BAP & $>=3$ & 0.668 & 0.28 \\
\hline
\end{tabular}




\section{DECAF SCORE}

A decision threshold of DECAF score $>2$ was found to have a sensitivity of $50 \%$ and specificity of $81 \%$, Accuracy of $73.3 \%$

Table 4: Sensitivity and specificity for different points of decaf

\begin{tabular}{|l|c|c|}
\hline Positive if Greater Than or Equal To & Sensitivity & Specificity \\
\hline-1.00 & 1.00 & 0.00 \\
\hline 0.50 & 1.00 & 0.24 \\
\hline 1.50 & 0.76 & 0.54 \\
\hline 2.50 & 0.50 & 0.81 \\
\hline 3.50 & 0.26 & 0.92 \\
\hline 5.00 & 0.00 & 1.00 \\
\hline
\end{tabular}

\begin{tabular}{|l|c|}
\hline \multicolumn{2}{|l|}{ Table 5: Statistical parameters of DECAF } \\
\hline Sensitivity & 50.0 \\
\hline Specificity & 81.3 \\
\hline False Negative & 50.0 \\
\hline False positive & 18.8 \\
\hline Positive Predictive value & 47.5 \\
\hline Negative Predictive value & 82.7 \\
\hline Positive Likelihood ratio & 2.7 \\
\hline Negative Likelihood ratio & 0.6 \\
\hline Accuracy & 73.3 \\
\hline
\end{tabular}

\section{DISCUSSION}

Despite improvements in care, death during hospitalization for AECOPD is a challenging issue $^{7}$ AECOPD being both common and often fatal, accurate prognostication of patients hospitalized with an exacerbation is important and difficult ${ }^{3}$.

In our study population of 150 cases of AECOPD, $38(25 \%)$ patients had a fatal outcome. This mortality rate matches with published figures; as it was published that in-hospital mortality of 4-30\% has been reported in patients with AECOPD requiring hospitalization ${ }^{[3,7,10,}$

Our study showed statistically significant relation of mortality with eosinopenia, changes in mentation, tachypnea, hyponatremia and hypoalbuminemia. There is also a statistically significant relation between higher grades of baseline dyspnea, higher Serum creatinine and mortality. Using DECAF 40 patients had score more than 2 and 110 had scores less than or equal to 2 . Out of 40 i.e., score $>2,19(47.5 \%)$ died. In patients with less scores only $17 \%$ died. Using BAP score there were 75 patients each with scores less than or equal to 1 and more than 1.In group with higher score there is higher mortality $(34.66 \%)$ compared to scores with low value(16\%) Using CURB -65 Score there are total 65 patients with scores more than 1.In that $40 \%$ died. In the less score group only $14.11 \%$ died.

Nafae et al. ${ }^{7}$ carried out a study on 200 AECOPD patients, they illustrated that the DECAF score showed an excellent discrimination for in-hospital mortality (AUROC =0.83). Furthermore, the DECAF Score performed significantly better in the prediction of in-hospital mortality than the Acute Physiology and Chronic Health Evaluation (APACHE) II prognostic index (AUROC $=0.68$, DECAF vs. APACHE II, $p=0.03$ ) and the COPD and Asthma Physiology Score (CAPS) (AUROC $=0.65, \mathrm{p}=0.01$ ); which have been proposed as useful predictive systems in AECOPD. They also found that the DECAF score was a significantly stronger predictor of in-hospital mortality than CURB-65 for a subgroup of patients with radiological consolidation (AUROC $=0.87$ vs. 0.65, $\mathrm{p}=0.02$ ). Comparing the AUROC of DECAF with CURB-65 \& APACHE-2 in our study, the differences were not statistically significant. The largest area under ROC was 0.729 for DECAF, followed by CURB-65 with 0.709 and APACHE 2 which had 0.700. CAPS score had an AUROC of 0.680 and BAP score had 0.668, which indicated they had poor accuracy in this setting. CURB 65 system which was initially developed for risk stratification in CAP, was compared with DECAF in this study and was found to be inferior to DECAF in prediction of in-hospital mortality for both patients with (AUROC 0.77 vs. 0.66, $\mathrm{p}=0.003, \mathrm{n}=299$ ) and without (AUROC $=0.87 \mathrm{vs}$. $0.72, \mathrm{p}=0.002, \mathrm{n}=621$ ) consolidation. In the current study AUROC for DECAF with consolidation=0.620, CURB with consolidation $=0.740$ $(\mathrm{p}=0.5)$. AUROC for DECAF without consolidation $=0.752, \quad \mathrm{CURB}=0.693 \quad(\mathrm{p}=0.234)$. Although AUROC for CURB with consolidation is higher than DECAF, the difference is not statistically significant. 
Zidan et al. ${ }^{11}$ tried to replace the atrial fibrillation item in the DECAF score (which has no significant value $(\mathrm{p}=0.618)$ ) to mortality by the frequency of admission (which has a significant relation to the mortality $(p<0.001)$, and they called the new score Modified DECAF score (dyspnea, eosinopenia, consolidation, respiratory acidosis and frequency of admission). There were significant values $(\mathrm{p}<0.001)$ between the modified DECAF score and mortality due to AECOPD. They concluded that the Modified DECAF score is more sensitive and more specific in predicting in-hospital mortality in acute exacerbation of COPD than the DECAF score with no significant difference between the two scores. ${ }^{11}$

The BAP-65 score developed by Shorr et al. ${ }^{12}$ depends on age, altered mental status, pulse and one laboratory marker which is the BUN. They found that it had AUROC $=0.77$ and it correlated well with multiple clinical outcomes ranging from in-hospital mortality and need for mechanical ventilation to length of stay and cost. They claimed that the BAP-65 score also identified subjects unlikely to need mechanical ventilation. 12. In our study BAP score had an AUROC of 0.682 , or poor accuracy.

The APACHE II is a severity of disease classification system, which uses 12 routine physiological variables and its increasing values from 0 to 71 signifies increasing risk of mortality $^{13}$. Different studies showed varying AUROC for APACHE II in AECOPD. The CAPS derivation study by Wildman et al, showed AUROC of $0.663^{14}$, The DECAF derivation study by Steer, Gibson et al showed an AUROC of 0.73 3 , our study showed an AUROC of 0.700, which was not significantly different from DECAF.

The CAPS score developed by Wildman et al in 2006 using eight variables, was found to have an AUROC of 0.718 in the derivation study; validation study showed similar results and discrimination was also found to be better than APACHE II. In the current study CAPS score had an AUROC of 0.680, which implied poor accuracy and was behind DECAF, CURB 65 and APACHE II.

The DECAF score was derived by Steer, Gibon et al in 2012 from a study population of 960, it underwent internal bootstrap validation and was found to have an AUROC of 0.86 (95\% CI 0.82 to 0.89 ) in the prediction of in-hospital mortality. In the current study it had an AUROC of 0.729 and was the highest among all scores tested. At a decision threshold of DECAF score $>2$ it could predict mortality with a sensitivity of $50 \%$ and specificity of $81 \%$.

\section{CONCLUSION}

It is recommended to use a scoring system for AECOPD in assessing mortality in the emergency setting and for guiding further treatment decisions. According to current study DECAF score (>=2) best correlates with in hospital mortality. Individual parameters that correlated best with mortality are Altered Mentation (GCS $<15)$, Respiratory rate $>30 / \mathrm{min}$, Baseline dyspnoea of eMRCD 5b, Elevated WBC count (>40000), Hyponatremia (S.Na+ $<130 \mathrm{mEq} / \mathrm{L}$, Hypoalbuminemia $(<3.5 \mathrm{~g} / \mathrm{dL})$ at the time of admission.

Other general scoring systems like CURB 65 and APACHE II can also predict mortality in AECOPD with an accuracy which is lesser than DECAF but without a statistically significant difference.

ACKNOWLEDGEMENT: Authors would like to thank Medical Records Department, Pariyaram Medical College for assistance in data collection, Dr. Oommen P Mathew for advice on statistical analysis.

LIMITATIONS: Single centre study.

\section{ETHICAL APPROVAL}

The study was approved by the Institutional Ethical Committee.

Source of Support: Nil

Conflict of Interest: None Declared. 


\section{REFERENCES}

1. GOLD- Global strategy for the diagnosis, management and prevention of Chronic Obstructive Pulmonary Disease

2. Yousif M, El Wahsh RA. Predicting inhospital mortality in acute exacerbation of COPD: Is there a golden score? Egypt J Chest Dis Tuberc. 2016;65(3):579-84.

3. Steer J, Gibson J, Bourke SC. The DECAF Score: predicting hospital mortality in exacerbations of chronic obstructive pulmonary disease. Thorax [Internet]. $2012 \mathrm{Nov}$ [cited 2016 Nov 21];67(11):970-6. Available from: http://www.ncbi.nlm.nih.gov/pubmed/22895999

4. Wildman MJ, Harrison DA, Welch CA, Sanderson C. A new measure of acute physiological derangement for patients with exacerbations of obstructive airways disease: The COPD and Asthma Physiology Score ARTICLE IN PRESS. Respir Med. 2007;101.

5. Zhang M, Zhao Y, Luo Y, Wang X, Yang $Y$, Lin $Y$. [The value of coexisting pneumonia and British Thoracic Society CURB-65 score in predicting early mortality rate in patients with acute exacerbation of chronic obstructive pulmonary disease]. Zhonghua Jie $\mathrm{He} \mathrm{He} \mathrm{Hu} \mathrm{Xi} \mathrm{Za}$ Zhi [Internet]. 2013 Apr [cited 2016 Nov 21];36(4):269-73. Available from: http://www.ncbi.nlm.nih.gov/pubmed/239 45340

6. Knaus WA, Draper EA, Wagner DP, Zimmerman JE. APACHE II: a severity of disease classification system. Crit Care Med [Internet]. 1985 Oct [cited 2016 Nov 22];13(10):818-29. Available from: http://www.ncbi.nlm.nih.gov/pubmed/392 8249

7. Nafae R, Embarak S, Gad DM. Value of the DECAF score in predicting hospital mortality in patients with acute exacerbation of chronic obstructive pulmonary disease admitted to Zagazig University Hospitals, Egypt. Egypt J Chest Dis Tuberc. 2015;64(1):35-40.0

8. Yousif $\mathrm{M}, \mathrm{El}$ Wahsh RA. Predicting inhospital mortality in acute exacerbation of COPD: Is there a golden score? Egypt J Chest Dis Tuberc. 2016;65(3):579-84.

9. S.P. Patil, J.A. Krishnan, N. Lechtzin, et al, In-hospital mortality following acute exacerbations of chronic obstructive pulmonary disease, Arch. Intern. Med. 163 (2003) 1180-1186.

10. A. Bustamante-Fermosel, J.M. De MiguelYanes, M. Duffort-Falco, et al, Mortalityrelated factors after hospitalization for acute exacerbation of chronic obstructive pulmonary disease: the burden of clinical features, Am. J. Emerg. Med. 25 (2007) 515-522.

11. M.H. Zidan, A.K. Rabie, M.M. Megahed, M.Y. Abdel-Khalek, The usefulness of the DECAF score in predicting hospital mortality in acute exacerbations of chronic obstructive pulmonary disease, Egypt. J. Chest Dis. Tuberculosis 64 (2015) 75-80

12. A.F. Shorr, X. Sun, R.S. Johannes, A. Yaitanes, Y.P. Tabak, Validation of a novel risk score for severity of illness in acute exacerbations of COPD, Chest 140 (5) (2011) 1177-1183

13. Knaus WA, Draper EA, Wagner DP, Zimmerman JE. APACHE II: a severity of disease classification system. Crit Care Med [Internet]. 1985 Oct [cited 2016 Nov 22];13(10):818-29. Available from: http://www.ncbi.nlm.nih.gov/pubmed/392 8249

14. Wildman MJ, Harrison DA, Welch CA, Sanderson C. A new measure of acute physiological derangement for patients with exacerbations of obstructive airways disease: The COPD and Asthma Physiology Score ARTICLE IN PRESS. Respir Med. 2007;101. 